\title{
CHARACTERIZATION OF COUNTERFEIT ARTESUNATE ANTIMALARIAL TABLETS FROM SOUTHEAST ASIA
}

\author{
KRYSTYN ALTER HALL, PAUL N. NEWTON, MICHAEL D. GREEN, MARLEEN DE VEIJ, PETER VANDENABEELE, \\ DAVID PIZZANELLI, MAYFONG MAYXAY, ARJEN DONDORP, AND FACUNDO M. FERNANDEZ* \\ School of Chemistry and Biochemistry, Georgia Institute of Technology, Atlanta, Georgia; Wellcome Trust-Mahosot Hospital-Oxford \\ Tropical Medicine Research Collaboration, Microbiology Laboratory, Mahosot Hospital, Vientiane, Lao People's Democratic \\ Republic; Centre for Clinical Vaccinology and Tropical Medicine, Churchill Hospital, Oxford University, Oxford, United Kingdom; \\ Division of Parasitic Diseases, National Center for Infectious Diseases, Centers for Disease Control and Prevention, Atlanta, Georgia; \\ Laboratory of Analytical Chemistry, Ghent University, Ghent, Belgium; Light Impressions International, Leatherhead, Surrey, United \\ Kingdom; Wellcome Trust-Mahidol University-Oxford Tropical Medicine Research Programme, Faculty of Tropical Medicine, \\ Mahidol University, Bangkok, Thailand
}

\begin{abstract}
In southeast Asia, the widespread high prevalence of counterfeits tablets of the vital antimalarial artesunate is of great public health concern. To assess the seriousness of this problem, we quantified the amount of active ingredient present in artesunate tablets by liquid chromatography coupled to mass spectrometry. This method, in conjunction with analysis of the packaging, classified tablets as genuine, substandard, or fake and validated results of the colorimetric Fast Red TR test. Eight (35\%) of 23 fake artesunate samples contained the wrong active ingredients, which were identified as different erythromycins and paracetamol. Raman spectroscopy identified calcium carbonate as an excipient in $9(39 \%)$ of 23 fake samples. Multivariate unsupervised pattern recognition results indicated two major clusters of artesunate counterfeits, those with counterfeit foil stickers and containing calcium carbonate, erythromycin, and paracetamol, and those with counterfeit holograms and containing starch but without evidence of erythromycin or paracetamol.
\end{abstract}

\section{INTRODUCTION}

Counterfeit drugs, as defined by the World Health Organization (WHO), are those that are "deliberately and fraudulently mislabeled with respect to identity and/or source ... with fake packaging, with the wrong ingredients, without active ingredients, or with insufficient active ingredient." ${ }^{1}$ Public health is at increasing risk because of an apparent growing global epidemic of the manufacture and trade of counterfeit pharmaceuticals. For example, in Haiti, India, Nigeria, and Bangladesh some 500 children died of acute renal failure after ingesting counterfeit paracetamol (acetaminophen) and cough syrup made using diethylene glycol, a renal toxin. ${ }^{2,3}$ Although there are few accurate estimates of the scale of the problem, only a few counterfeit drug incidents are reported to the appropriate enforcement agencies; thus, numbers of those affected by counterfeit drugs are likely to be grossly underestimated. ${ }^{4,5}$

Since the late 1990s, counterfeit drugs manufactured to mimic antimalarial medicines have been detected in increasing numbers. ${ }^{6-8}$ Each year, 300-500 million people in Asia and Africa contract Plasmodium falciparum malaria and approximately 1.5 million die. ${ }^{9}$ The control of malaria, which is dependent on effective antimalarial drugs and bed nets, has been severely hampered by a widespread increase in the prevalence of drug-resistant malaria parasites. ${ }^{10}$ Artesunate, an artemisinin derivative, is widely and increasingly used in the treatment of $P$. falciparum malaria in many southeast Asian and African countries and is vital for the therapy of drug-resistant malaria. ${ }^{11}$ In Asia, artesunate has become the target of an extremely sophisticated and prolific counterfeit drug trade that includes the counterfeiting of both the arte-

\footnotetext{
* Address correspondence to Facundo M. Fernandez, School of Chemistry and Biochemistry, Georgia Institute of Technology, 770 State Street, Atlanta, GA 30332. E-mail: facundo.fernandez@ chemistry.gatech.edu
}

sunate tablets and packaging, which look extremely similar to the authentic product. ${ }^{12-15}$ In response to this public health problem, Green and others developed a colorimetric Fast Red TR dye test ${ }^{16}$ that rapidly and inexpensively screens for the presence of artemisinin-derived compounds such as artemether, artesunate, and dihydroartemisinin in tablets. There are at least 10 different manufacturers of artesunate tablets in Asia. The tablets produced by them have a stated artesunate content of $50 \mathrm{mg}$. Surveys conducted in 1999-2000 and 20012002 in Cambodia, Lao People's Democratic Republic (Laos), Burma (Myanmar), on the Thailand/Burma border, and Vietnam demonstrated that $38 \%$ and $53 \%$, respectively, of artesunate tablets contained no active ingredient. ${ }^{6,13}$ To date, only artesunate labeled as made by Guilin Pharmaceutical Co., Ltd. (Guilin, Guangxi, People's Republic of China) has been found to be counterfeit. Visual inspection, including the examination of the holograms, bar codes, printing, crimping, color, size, weight, and consistency of the putative artesunate tablets, was in good agreement with the colorimetric test results in the first survey, but not in the second survey. The counterfeiters appear to have responded to the increase in public awareness by producing more sophisticated counterfeit holograms and packaging, making it very difficult to distinguish the counterfeit and genuine drugs. ${ }^{14,15}$

Little is known about the chemical composition of these counterfeit medicines, the potential presence of toxic substances, or the fake artesunate production sources. Therefore, we developed a liquid chromatography-mass spectrometry (LC-MS) method to quantitatively evaluate the contents of artesunate tablets, to validate the Fast Red TR colorimetric test results, and to investigate the presence of other active ingredients. Raman spectroscopy was used to characterize the excipients present in the tablets and complement the LC-MS analysis. We then used multivariate pattern recognition methods to examine the chemical similarities and differences between the chemical fingerprints of different types of fake tablets, and to correlate these similarities with packaging characteristics and sample origin. 


\section{MATERIALS AND METHODS}

Artesunate (CAS \# 88495-63-0) and erythromycin, (CAS \# 114-07-8) were obtained from Apin Chemicals Ltd. (Abingdon, United Kingdom) and Fluka Biochemica (Buchs, Switzerland), respectively. Artesunate standards were prepared in high performance liquid chromatography (HPLC) grade methanol (Sigma Aldrich, St. Louis, MO). Erythromycin standards were prepared in a 50/50 solution (v/v) of HPLC grade acetonitrile (Sigma Aldrich) and pure water (Barnstead International, Dubuque, IA). Fresh standards were prepared on a daily basis from stock solutions that were stored at $4{ }^{\circ} \mathrm{C}$.

Sample collection and preparation. A subset of 34 counterfeit and genuine artesunate tablets from two surveys ${ }^{6,13}$ representing the diversity of different packaging types recognized in 2004 (see supplementary material in the report by Newton and others ${ }^{15}$ ) were selected for LC-MS . Tablets were crushed with a mortar and pestle and thoroughly homogenized; $100 \mathrm{mg}$ of sample were suspended in $10 \mathrm{~mL}$ of a 50/50 acetonitrile/water ( $\mathrm{v} / \mathrm{v})$ mixture and extracted on a rotary shaker for two hours. All samples and standards were filtered through a $0.45-\mu \mathrm{m}$ polytetrafluoroethylene membrane filter (Pall Corporation, Ann Arbor, MI). Tablets and tablet extracts were kept refrigerated $\left(4^{\circ} \mathrm{C}\right)$ until analysis. After preliminary LC-MS runs, samples were diluted with methanol or 50/50 acetonitrile/water (v/v) as needed.

Characteristics of the packaging of fake artesunate tablets. Fourteen different types of counterfeit artesunate tablet samples, based on the packaging appearance and holograms, have been described (see supporting information and Table $\mathrm{S} 1$ in the report by Newton and others ${ }^{15}$ ). Type 1 counterfeit artesunate bears no sticker or hologram, types $2,5,8,11$, and 12 bear foil stickers imitating the genuine hologram, and types $3,4,6,7,9,10,13$, and 14 bear hologram copies of the genuine holograms.

Tablet testing by LC-MS. An introduction to LC-MS for pharmaceutical analysis can be found elsewhere. ${ }^{17}$ All samples were analyzed in a blind fashion to avoid any possible bias in the analysis. The LC was performed on an Agilent 1100 system equipped with a solvent degasser, a binary pump, a thermostated column compartment (held at $25^{\circ} \mathrm{C}$ ), an autosampler, and a diode array detector. A 2.1-mm internal diameter, 150-mm long Zorbax Extend-C18 column (Agilent, Palo Alto, CA) equipped with a precolumn was used. The LC was operated at a flow rate of $200 \mu \mathrm{L} /$ minute, with an injection volume of $20 \mu \mathrm{L}$. The binary pump used HPLC grade water with $0.01 \%$ acetonitrile as mobile phase A and acetonitrile as mobile phase B. For tablet fingerprinting, the LC gradient started at $5 \% \mathrm{~B}$ and ramped to $45 \% \mathrm{~B}$ until 7 minutes, then increasing to $100 \% \mathrm{~B}$ from 7 to 8 minutes and held at $100 \%$ B until 12 minutes. The system was set with a fourminute post-run time to equilibrate the column to the original mobile phase composition. To ensure maximum reproducibility in fingerprinting, all the organic extracts obtained from fake tablets where run on the same day and by triplicate. Blank runs were inserted between samples to eliminate carryover. Artesunate was quantified as follows: HPLC grade water with $0.01 \%$ methanol was used as mobile phase A and methanol as mobile phase B. The LC solvent gradient started at $35 \% \mathrm{~B}$ for 3 minutes, and then ramped to $100 \% \mathrm{~B}$ in 18 minutes, where it was held for 30 minutes. It was then ramped back down to $35 \% \mathrm{~B}$ in 15 minutes. The retention time for artesunate was 18.1 minutes.

The LC system was coupled to an AccuTOF time-of-flight (TOF) mass spectrometer (JEOL, Peabody, MA) via an orthogonal electrospray interface operating at $2 \mathrm{kV}$. Although negative ion mode electrospray ionization would seem the first choice for artesunate analysis because of its carboxylic acid functionality, positive ion mode also produces sufficient sensitivity, and ensures detection of other compounds that might be present in the sample. The ammonium $\left[\mathrm{M}+\mathrm{NH}_{4}\right]^{+}$ $(m / z=402)$ and sodium $[\mathrm{M}+\mathrm{Na}]^{+}(m / z=407)$ artesunate adduct ions were used for quantitation purposes. The MS system allowed for accurate mass measurements with an average mass accuracy of $2 \mathrm{ppm}$ at $m / z=609$. The NIST mass spectral library (version 2.0 a) was used to search elemental formulas of unknown compounds. ${ }^{18}$

Erythromycin was quantified by triplicate injections using flow injection-MS as follows: a Vici Cheminert M6-liquid handling pump (Valco Instruments Co. Inc. Houston, TX) operated at $200 \mu \mathrm{L} /$ minute was connected to a manual sixway HPLC injection valve equipped with a $20-\mu \mathrm{L}$ loop (Valco Instruments Co. Inc.). The mobile phase consisted of $50 \%$ acetonitrile in water. The area of the mass-selected chromatographic peaks was integrated producing a linear calibration curve between $2 \mathrm{nM}$ and $145 \mathrm{nM}$.

Tablet testing by Raman spectroscopy. The analyses were carried out on a Renishaw System-1000 spectrometer (Renishaw, Wotton-under-Edge, United Kingdom) that was connected to an Olympus (Center Valley, PA) BH-2 microscope. The diode laser has a power of $50 \mathrm{~mW}$ at the source and a wavelength of $785 \mathrm{~nm}$ (DL 100; TuiOptics GmbH, Martinsried/Munich, Germany). The collected Raman radiation was dispersed with a 1,200 lines/mm grating and focused on a Peltier-cooled CCD detector (Renishaw). All spectra were recorded in the spectral window of 200 to $1800 / \mathrm{cm}$, with 5 accumulations of 30 seconds. Vankeirsbilck and others provide an interesting review on the use of Raman spectroscopy for pharmaceutical analyses. ${ }^{19}$

Data analysis. Time-resolved mass spectra were exported from the AccuTOF instrument in ASCII format, resulting in 44 spectra, each one averaged over a 16.4-second retention time interval. All LC-MS data was processed in Matlab version 7.0 (The MathWorks, Natick, MA). After being exported as ASCII files, each LC-MS run produced a 44 by 18,001 data point matrix. The three matrices obtained for each triplicate injection of identical sample extracts were averaged and prepared for multivariate analysis by unfolding them into a single $44 \times 18,001=792,044$ element vector. This vector contained, in one row, all mass spectra ordered by increasing retention times. The vectors corresponding to the 23 fake artesunate tablet samples were then combined into a single 23 by 792,044 matrix that was analyzed using K-nearest neighbor (KNN) hierarchical clustering. ${ }^{20}$

\section{RESULTS}

Artesunate content. The mean (95\% confidence interval) artesunate content of artesunate tablets collected in Vietnam, Burma, Thailand, Laos and Cambodia that tested positive in the Fast Red TR colorimetric test was 55 (51-59) mg, except for one tablet furnished with the genuine Guilin Pharmaceutical Co., Ltd. hologram, which was collected in Siem Reap 
TABLE 1

Artesunate content for tablets that tested positive in colorimetric tests*

\begin{tabular}{|c|c|c|}
\hline Sample code & Sample collection place & $\begin{array}{c}\text { Artesunate content } \\
(\mathrm{mg} / \text { tablet } \pm \mathrm{SD})\end{array}$ \\
\hline $5 \dagger$ & Mae La, Thailand/Burma border & $54 \pm 1$ \\
\hline $18 \ddagger$ & Ho Chi Minh City, Vietnam & $51 \pm 6$ \\
\hline $31 \S$ & Ho Chi Minh City, Vietnam & $54 \pm 5$ \\
\hline $32 \dagger$ & Mueang Feuang, Laos & $49 \pm 6$ \\
\hline $39 \dagger$ & Myitkyina, Burma & $42 \pm 12$ \\
\hline 12064I & Sekong, Laos & $62 \pm 4$ \\
\hline $12065 \pi$ & Sekong, Laos & $57 \pm 3$ \\
\hline $12068 \mathbb{1}$ & Attepeu, Laos & $65 \pm 14$ \\
\hline $13008+$ & Siem Reap, Cambodia & $21 \pm 2$ \\
\hline $13013 \dagger$ & Ko Kong, Cambodia & $58 \pm 3$ \\
\hline 63001\# & Phnom Phenh, Cambodia & $56 \pm 2$ \\
\hline
\end{tabular}

(Cambodia) and contained $21 \mathrm{mg}$ of artesunate (Table 1). This tablet was therefore considered substandard because it does not meet the quality specifications set by this product by the legitimate manufacturer. ${ }^{21}$ It was collected 13 months after date of manufacture as stated on the blisterpacket.

Artesunate tablets that tested negative by the Fast Red dye test were all labeled as being manufactured by Guilin Pharmaceutical Co. Ltd. (Guilin, Guangxi, People's Republic of China). Artesunate was not detectable in these tablets by LC-MS (limit of detection $<1 \mu \mathrm{g}$ ) (Table 2). Overall, 23 $(68 \%)$ of 34 artesunate samples did not contain the expected active ingredient and there was $100 \%$ agreement between the Fast Red dye test results and the LC-MS results.

Identification of unexpected ingredients. During artesunate quantitation, samples 12050, 12052, 12053, 12054, 12057, 12060, 12061, and 12062 (Table 2) had similar intense peaks in the $700-750 \mathrm{~m} / \mathrm{z}$ range that could not be assigned to any artesunate dimer ions. Accurate mass analysis was performed on sample 12053 and showed an exact mass value of 734.4703 Da for the monoisotopic peak of the suspected ingredient, with an elemental composition of $\mathrm{C}_{37} \mathrm{H}_{67} \mathrm{NO}_{13}$. A search within the NIST mass spectral database ${ }^{18}$ returned a match corresponding to the antibiotic erythromycin A with a theoretical mass of $734.4691 \mathrm{Da}$. The total ion chromatograms of an erythromycin standard and of sample 12053 showed matching retention times of 13.38 and 13.55 minutes, respectively. The fragmentation patterns for sample and standard were also identical. $^{22}$ The relative abundance of the $[\mathrm{M}+\mathrm{H}]^{+}$isotopic peaks was also examined and was successfully matched to the erythromycin standard, further confirming the identity of this unexpected ingredient. Fake artesunate tablets contained a total amount of erythromycin A that ranged from 20 to 191 $\mathrm{mg} / \mathrm{g}$ of tablet (Table 2).

Erythromycin formulations generally contain more than one type of erythromycin, with erythromycin A being the most abundant. ${ }^{23}$ Inspection of the erythromycin spectral region showed several additional signals. Accurate mass measurements were performed on all these peaks, and the following erythromycins were identified: erythromycin B (theoretical $\mathrm{m} / z=718.4714 \mathrm{Da}$, observed $\mathrm{m} / z=718.4742 \mathrm{Da}$ ); erythromycin $\mathrm{C}$ (theoretical $\mathrm{m} / z=720.4534 \mathrm{Da}$, observed $\mathrm{m} / \mathrm{z}=720.4547$ ); erythromycin $\mathrm{E}$ (theoretical $\mathrm{m} / \mathrm{z}=$

TABLE 2

Sample origin and type of fake packaging sticker/holograms for tablets which tested negative in colorimetric tests*

\begin{tabular}{|c|c|c|c|c|c|}
\hline Sample code $\dagger$ & Sample collection place & Fake hologram type & $\begin{array}{l}\text { Erythromycin A content } \\
(\mathrm{mg} / \mathrm{g} \text { tablet } \pm \mathrm{SD})\end{array}$ & Paracetamol & $\begin{array}{l}\text { Excipient detected by } \\
\text { Raman spectroscopy }\end{array}$ \\
\hline \multicolumn{6}{|c|}{ Counterfeit artesunate with foil sticker imitations of genuine holograms } \\
\hline 12054 & Pakse, Laos & 5 & $27 \pm 4$ & No & $\mathrm{CaCO}_{3}$ \\
\hline 12061 & Salavan, Laos & 5 & $191 \pm 7$ & Yes & Not identified \\
\hline 12057 & Pakse, Laos & 8 & $58 \pm 1$ & No & $\mathrm{CaCO}_{3}$ \\
\hline 12050 & Pakse, Laos & 8 & $50 \pm 1$ & No & $\mathrm{CaCO}_{3}$ \\
\hline 12060 & Salavan, Laos & 8 & $<1 \dagger$ & Yes & Not identified \\
\hline 12058 & Pakse, Laos & 8 & Not detected & No & $\mathrm{CaCO}_{3}$ \\
\hline 12052 & Pakse, Laos & 11 & $57 \pm 5$ & Yes & $\mathrm{CaCO}_{3}$ \\
\hline 12062 & Salavan, Laos & 11 & $20 \pm 5$ & Yes & Not identified \\
\hline 12053 & Pakse, Laos & 5 & $<1 \dagger$ & Yes & Not identified \\
\hline 13 & Myitkyina, Burma & 1 & Not detected & No & Starch \\
\hline 980201 & Ho Chi Minh City, Vietnam & 1 & Not detected & No & $\mathrm{CaCO}_{3}$ \\
\hline 29 & Ho Chi Minh City, Vietnam & 2 & Not detected & No & $\mathrm{CaCO}_{3}$ \\
\hline 97 & Ho Chi Minh City, Vietnam & 2 & Not detected & No & $\mathrm{CaCO}_{3}$ \\
\hline \multicolumn{6}{|c|}{ Counterfeit artesunate with hologram imitations of the genuine holograms } \\
\hline 12051 & Pakse, Laos & 3 & Not detected & No & Starch \\
\hline $13011 / 1$ & Anlong Veng, Cambodia & 3 & Not detected & No & Starch \\
\hline $13011 / 2$ & Anlong Veng, Cambodia & 3 & Not detected & No & Starch \\
\hline 12055 & Pakse, Laos & 3 & Not detected & No & Starch \\
\hline 12071 & Paksong, Laos & 3 & Not detected & No & Starch \\
\hline 12063 & Salavan, Laos & 3 & Not detected & No & Starch \\
\hline 12067 & Attepeu, Laos & 3 & Not detected & No & Starch \\
\hline 12056 & Pakse, Laos & 4 & Not detected & No & Starch \\
\hline 12070 & Paksong, Laos & 4 & Not detected & No & Starch \\
\hline 13007 & Siem Reap, Cambodia & Cutoff & Not detected & No & $\mathrm{CaCO}_{3}$ \\
\hline
\end{tabular}

* The classification of the fake stickers/holograms is detailed under supporting information. Artesunate was not detected in any of these samples. The printing on the blisterpack of specimen 13007 was unclear, suggesting that it was counterfeit. The presence of calcium carbonate suggests that it would have carried a foil sticker. All samples were labeled as if being manufactured by Guilin Pharmaceutical Co. Ltd. (People's Republic of China).

$\dagger$ Erythromycin A was detected, but the signal was too weak for accurate quantification. 


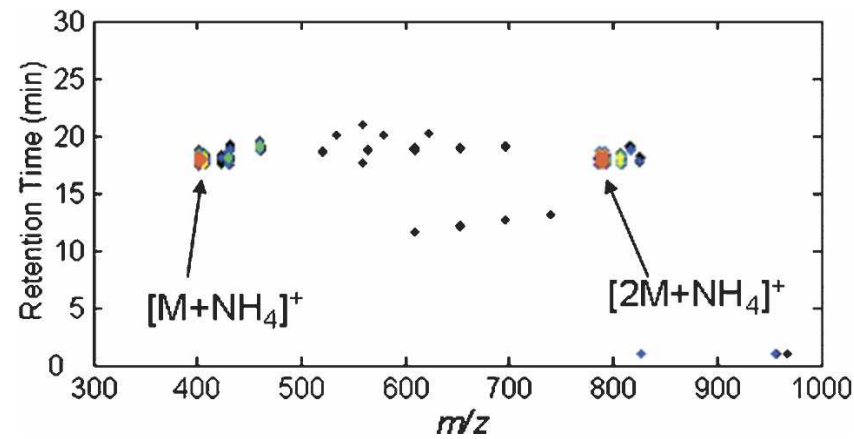

FIGURE 1. Chemical fingerprint plot of a genuine artesunate tablet. $\mathrm{M}=$ artesunic acid. Larger red dots denote the most intense signals and black small dots represent the weakest ones. Signals with intermediate intensity were coded according to the color scale yellow $>$ green $>$ cyan $>$ blue. This figure appears in color at www.ajtmh.org.
748.4483 Da, observed $m / z=748.4503 \mathrm{Da}$ ); erythromycin $\mathrm{F}$ (theoretical $\mathrm{m} / z=750.4640 \mathrm{Da}$, observed $\mathrm{m} / \mathrm{z}=750.4633$ Da), and anhydroerythromycin (theoretical $\mathrm{m} / \mathrm{z}=716.4585$ Da, observed $\mathrm{m} / z=716.4576 \mathrm{Da})$. Only erythromycin A was detected in sample 12053 (Table S6).

Multivariate clustering of LC-MS chemical fingerprints. To investigate the relationship between samples geographic origin, the content and type of erythromycins present and the type of fake holograms observed on the packaging, multivariate pattern recognition was performed on the LC-MS data. Figure 1 shows the chemical fingerprint obtained by LC-MS for a genuine Guilin Pharmaceutical Co., Ltd. artesunate tablet. Two intense peaks with a retention time of approximately 18 minutes are observed. The most intense signals (red) correspond to the artesunate $\left[\mathrm{M}+\mathrm{NH}_{4}\right]^{+}$and $\left[2 \mathrm{M}+\mathrm{NH}_{4}\right]^{+}$ adduct ions (at $402 \mathrm{Da}$ and $786 \mathrm{Da}$, respectively). Figure 2

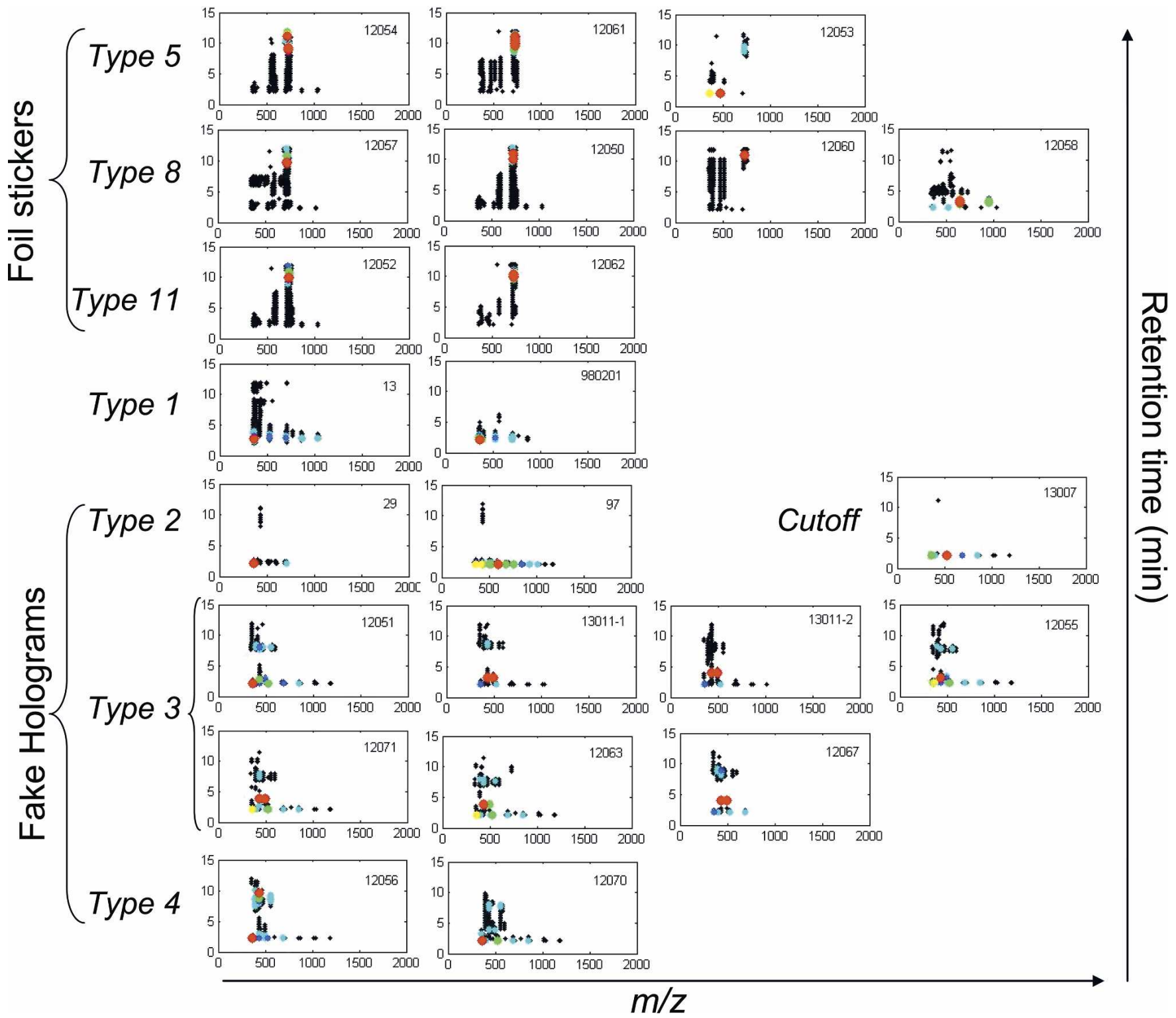

FIGURE 2. Chemical fingerprint plots of fake artesunate tablets. Sample code is indicated within each plot. The $\mathrm{x}$-axis represents $\mathrm{m} / \mathrm{z}$ values and the y-axis represents chromatographic retention time. This figure appears in color at www.ajtmh.org. 
shows contour plots of the LC-MS chemical fingerprints of 23 fake artesunate tablets. Comparison of Figures 1 and 2 clearly demonstrates that none of the fake artesunate samples resembles the genuine product in terms of chemical composition. However, several similarities among the fakes can be readily found by visual inspection of their fingerprints. Samples 12050, 12052, 12054, 12057, 12060, 12061, and 12062, which were collected between December 2002 and January 2003, show similar fingerprints because of the presence of erythromycins. Because of its lower erythromycin A content, sample 12053 presented a different fingerprint than the aforementioned samples. Raman spectroscopic analysis showed that samples 12050, 12052, 1205412057 , and 12058 had a common excipient signature, which was confirmed to be $\mathrm{CaCO}_{3}$, a common inorganic filler (Allen and others ${ }^{24}$ ). Genuine tablets use maize starch, not $\mathrm{CaCO}_{3}$, as the excipient. Samples 12060, 12061, and 12062 contained erythromycin, but no $\mathrm{CaCO}_{3}$ was detected. Calcium carbonate was also detected by Raman spectroscopy in samples 980201, 29, and 97 from Vietnam and 13007 from Cambodia.

The chemical signatures of samples 13011/1 and 13011/2 from Along Veng, Cambodia are similar. These tablets come from two different, but physically identical blister packs, that were bought at the same time from the same shop. The lack of refrigerated storage of the samples in shops and pharmacies in the seasonally high ambient temperatures in southeast Asia (up to approximately $40^{\circ} \mathrm{C}$ ) may be the cause of the small differences between the samples' fingerprints. These two samples showed a similar polymeric peak eluting at 2 minutes, spaced by $162 \mathrm{Da}$, which corresponds to a hexose unit. These signals originate from low molecular weight starch-related excipients ${ }^{24}$, which were also detected by Raman spectroscopy. Samples 12051, 12055, 12056, 12063, 12070, and 12071 showed fingerprints similar to samples 13011-1 and 13011-2, also corresponding to a starch-related excipient.

Figure 3A shows the KNN dendrogram of the counterfeit artesunate tablets' LC-MS data. Samples 12054, 12061, 12057 , $12052,12050,12062$, and 12060 are clustered towards the top portion of the dendrogram in a nested fashion. All these samples were collected in different parts of southern Laos and showed intense erythromycin signals. The ordering of these samples in the dendogram followed their erythromycin A content (Table 2), except for sample 12054 (containing $27 \mathrm{mg}$ of erythromycin $\mathrm{A} / \mathrm{g}$ tablet), which was placed at the top of the diagram. The fact that sample 12054 seems to be chemically unique is probably related to its different proportion of anhydroerythomycin A, a hydrolysis product of erythromycin A (Table S6), which indicates that a different (probably older) batch of degraded erythromycin may have been used.

The sample grouping in the dendogram based on chemical composition is also correlated with differences in the counterfeit types as judged by the packaging. For example, samples 12054 and 12061, which were placed in adjacent branches of the dendogram, showed the same type of rainbow foil sticker (type 5, not a true hologram but a sticker, see S1 in the report by Newton and others ${ }^{15}$ ) and were collected in nearby Lao cities (Pakse and Salavan). These two samples may come from separate batches that originated in a common production source using unsophisticated fake stickers. However, accurate mass MS experiments in the low molecular weight region showed that paracetamol (acetaminophen) had been added to sample 12061, but not to sample 12054
(Table 2). Samples 12057, 12052, and 12050, which were also obtained in Pakse, had type 8 or 11 fake foil stickers but are chemically different from samples 12054 and 12061 in that they all contain approximately $50 \mathrm{mg}$ of erythromycin-A/g. Samples 12052 and 12050 are further clustered, on the basis of their chemical composition, because of their higher relative percentage of erythromycin $\mathrm{A}$ in comparison to sample 12057.

Samples 12062 and 12060 were both collected in Salavan, Laos and had rainbow foil stickers (types 11 and 8, respectively) similar to samples 12057, 12052, and 12050. Their erythromycin fingerprint is similar (between $80 \%$ and $90 \%$ erythromycin A) but they differ in their absolute erythromycin content. Raman and LC-MS also showed the presence of paracetamol in both samples. Sample 12058 from Pakse (type 8 ), did not contain erythromycin, and seems to be placed between the Laos samples containing erythromycins with fake foil stickers and the remainder of the samples, which do not contain erythromycins and are affixed with more elaborate fake holograms.

The analysis of the similarities among samples without erythromycins (Table 2) showed interesting trends (Figure 3B). Sample 13, which was collected in Myitkyina, Burma in 2000, had no fake holograms or stickers present on the packaging (type 1). This sample is the most isolated from the rest of the samples from a geographic point of view, but it showed a Raman excipient signature consistent with starch similar to two samples with type 3 fake holograms: samples 12051 (Pakse, southern Laos) and 12071 (Paksong, southern Laos), collected in December 2002 and January 2003, respectively. Sample 980201, which was collected in Ho Chi Minh City, also had no fake sticker/hologram (type 1), but bore atypically large printing font on the blisterpack and a simple chemical fingerprint that partially resembled the fingerprint from Vietnam sample 29, which was affixed with a type 2 fake foil sticker. These two samples contained $\mathrm{CaCO}_{3}$ and might have been produced by the same manufacturing source, which added a fake foil sticker to its product in response to increased quality assurance measures put in practice by the genuine manufacturer.

Samples with fake holograms type 3 and 4 are grouped together at the bottom of the dendogram (Figure 3B). Samples 12071 and 12063 seem to be almost identical chemically, with matching LC-MS fingerprints. Their packaging showed a type 3 fake hologram and Raman analysis indicated the presence of starch in both samples. Sample 12070, which was collected in Paksong, was chemically similar to samples 12071 and 12063, but showed a more sophisticated type 4 fake hologram. Samples 12051, 13011, 12056, and 12055 were collected in Pakse, Laos and Anlong Veng, Cambodia (150 miles away) and appear to be chemically related and similar to the remainder of the samples detailed in Figure 3B. Sample 12056 showed a sophisticated type 4 fake hologram that was similar to that found on sample 12070 .

\section{DISCUSSION}

Between 1982 and 1999, 771 reports of counterfeit medicines were received by $\mathrm{WHO}, 48.4 \%$ of which were from the Western Pacific region, with most $(51.2 \%)$ labeled as antiinfectives. ${ }^{7}$ However, only $5-15 \%$ of the 191 WHO member 

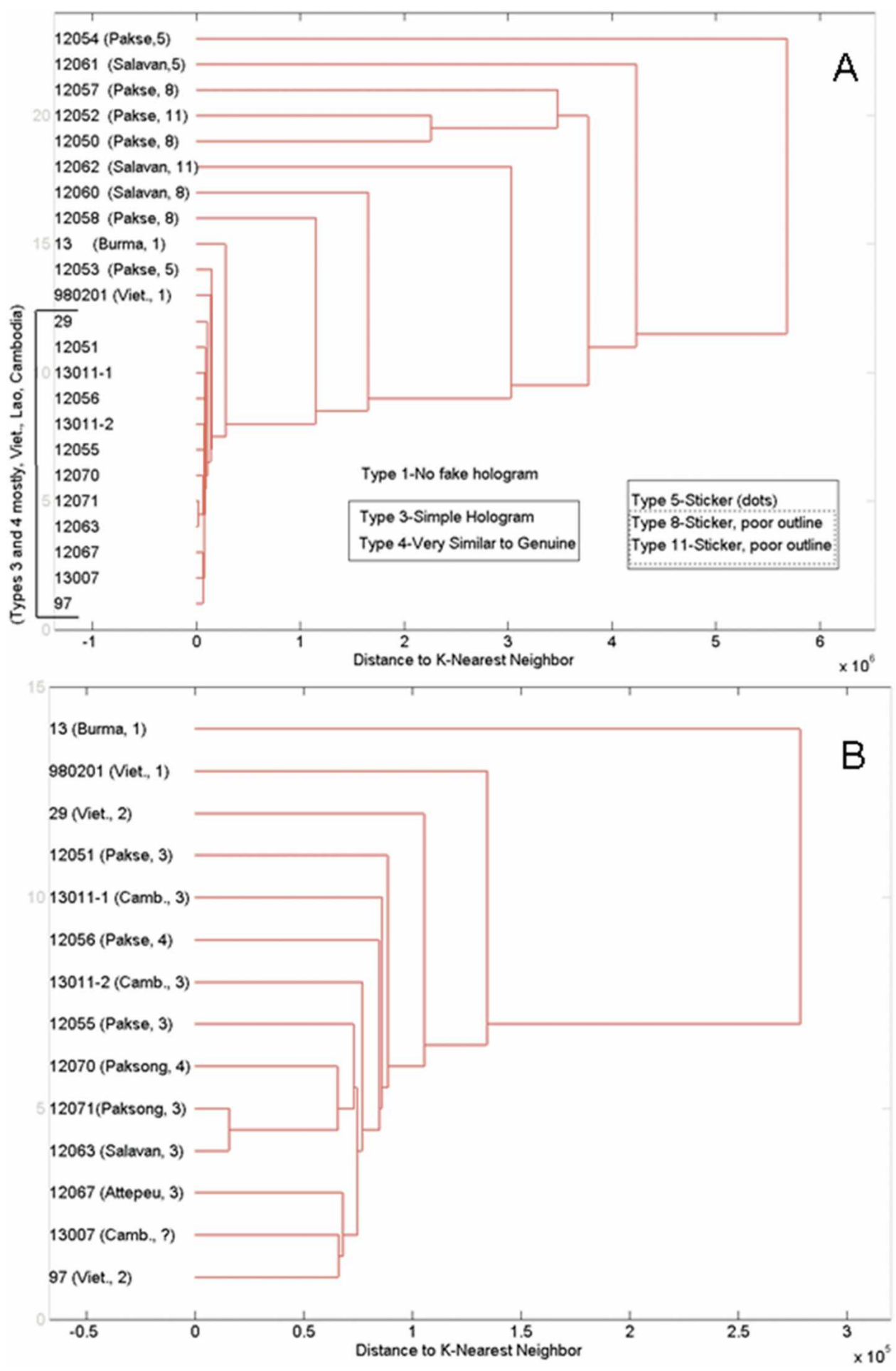

Figure 3. A, K-nearest neighbor dendogram showing similarities between fake artesunate tablets. B, Detail of the samples not containing erythromycins. Viet. = Vietnam; Camb. = Cambodia. This figure appears in color at www.ajtmh.org.

states report cases of counterfeit $\mathrm{drug}^{7}$ and the "scale of the problem ... is shrouded in ignorance, confusion and denial." 25 Antimalarials, among the most widely used drugs in tropical countries, seem to have been particularly targeted by counterfeiters. Of the 12 major antimalarial drugs used in the world today, there are recent reports of counterfeits of $8 .^{7}$ Counterfeit mefloquine and artesunate were first noticed in Cambodia in 1998 when suspiciously inexpensive tablets were found. ${ }^{26}$ A wide range of different counterfeit artesunate types, based on packaging and fake holograms, have been described and counterfeit artesunate has recently been found in Yunnan, China. ${ }^{27}$ Artesunate tablets, such as sample 13008 from Cambodia, containing subtherapeutic amounts of artesunate, whether they are counterfeit, substandard, or of reduced active ingredient because of poor storage, pose a great danger to public health because they can create an environ- 
ment that selects drug-resistant parasite strains. ${ }^{10,28}$ The instability of some drugs kept in inadequate storage conditions in tropical climates sometimes confounds drug quality assessment and detection of substandard drug samples. For this reason, it is not possible to assert if the insufficient levels of artesunate observed in sample 13008 are a product of inadequate storage or inadequate manufacturing practices. As shown in Table 1, samples collected prior to sample 13008, such as samples 5, 18, 31, 32, and 39, contained the expected amount of artesunate. Thus, we conclude that no noticeable sample degradation was observed while stored in our facilities. Alarmingly, small quantities of artesunate, which were probably added to the preparations with the intention to deceive the rapid colorimetric tests currently in use to detect counterfeits, ${ }^{15,29}$ have also been recently detected in counterfeit antimalarial tablets packaged with fake holograms.

The second major anomaly observed, the presence of the wrong active ingredients, is not uncommon in counterfeit anti-infective drugs. Some of the early counterfeit artesunate (collected in or before 1999) contained chloroquine that may have been added to give the counterfeits a bitter taste because there is a tradition in southeast Asia that antimalarials should be bitter. ${ }^{26}$ However, chloroquine has very low antimalarial efficacy throughout mainland southeast Asia and has been abandoned as therapy for $P$. falciparum malaria. ${ }^{10}$ Recent examples of unexpected ingredients in counterfeit antiinfective drugs include a sulfonamide drug (sulfamethazine or sulfisomidine) in counterfeit halofantrine syrup from west Africa $^{30}$ and chloramphenicol, metronidazole, pyrimethaminesulfadoxine, and metamizole in types of counterfeit artesunate not examined in this study. ${ }^{29}$ These potentially dangerous pharmaceuticals, with no or minimal antimalarial action, are probably leftover ingredients available to the counterfeiters in a poor quality production process.

Examination of the relationship between chemical composition and packaging suggests two major clusters of artesunate counterfeits, those with counterfeit foil stickers and containing $\mathrm{CaCO}_{3}$, erythromycin, and paracetamol, and those with counterfeit holograms and containing starch but without chemical evidence for the wrong active ingredients. In addition, of the two type 1 samples, one contained starch and one contained $\mathrm{CaCO}_{3}$, suggesting that the so-called type 1 counterfeit artesunate are a mixture of different recipes classified together in error because of the lack of external packaging clues.

That fake artesunate tablets prepared with different recipes have been found in groups of neighboring cities either indicates a common source of these fakes or a few sources (probably two or three) sharing a common distribution network. Fake artesunate tablets of identical types are found in diverse countries, with no clear spatial segregation into types, which suggests a counterfeiting operation with a widespread distribution network that extends across many porous borders. This criminal counterfeiting operation has been flooding the market with fake artesunate during the last eight years with inevitable consequences in terms of increased malaria mortality and morbidity. ${ }^{15}$

In summary, this study confirms the existence of fake artesunate tablets with no detectable active ingredient, describes the presence of one sample of sub-standard artesunate bearing the manufacturer's genuine hologram, and reports the detection of different erythromycins and paracetamol in fake artesunate. There was $100 \%$ agreement between the LC-MS results and those obtained using the rapid, inexpensive colorimetric artesunate Fast TR dye test. The results also suggest that a relationship between sample origin, chemical composition, and packaging exists.

Note: Supplemental Figures S1-S5 and supplemental Table S6 appear online at www.ajtmh.org.

Received March 4, 2006. Accepted for publication June 11, 2006.

Acknowledgments: We are extremely grateful to Professors Nicholas White and Nicholas Day and all who assisted in the collection of samples.

Financial support: Analysis of antimalarial drug samples was supported by a starter grant from the Society of Analytical Chemists of Pittsburgh (United States) to Facundo M. Fernandez. Collection of artesunate samples was supported by the Wellcome Trust of Great Britain as part of the Wellcome Trust-South East Asian Oxford Tropical Medicine Research Collaborations.

Authors' addresses: Krystyn Alter Hall and Facundo M. Fernandez, School of Chemistry and Biochemistry, Georgia Institute of Technology, 770 State Street, Atlanta, GA 30332, Telephone: 404-385-4432, Fax: 404-385-6447, E-mails: krystyn.hall@gmail.com and facundo.fernandez@chemistry.gatech.edu. Paul N. Newton, Wellcome Trust-Mahosot Hospital-Oxford Tropical Medicine Research Collaboration, Microbiology Laboratory, Mahosot Hospital, Vientiane, Lao PDR and Centre for Clinical Vaccinology and Tropical Medicine, Churchill Hospital, University of Oxford, Oxford, United Kingdom. Michael D. Green, Division of Parasitic Diseases, National Center for Infectious Diseases, Centers for Disease Control and Prevention, 1600 Clifton Road, Mailstop F12, Atlanta, GA 30333, E-mail: mdg4@cdc.gov. Marleen de Veij and Peter Vandenabeele, Laboratory of Analytical Chemistry, Ghent University, Proeftuinstraat 86, B-9000 Ghent, Belgium, E-mail: peter .vandenabeele@UGent.be. David Pizzanelli, Light Impressions International, 5 Mole Business Park 3, Randalls Road, Leatherhead, Surrey KT22 7BA, United Kingdom, E-mail: david@pizzanelli.wanadoo .co.uk. Mayfong Mayxay, Wellcome Trust-Mahosot Hospital-Oxford Tropical Medicine Research Collaboration, Microbiology Laboratory, Mahosot Hospital, Vientiane, Lao PDR, and Department of Post Graduates and Research, Faculty of Medical Science, National University of Laos, Lao PDR. Arjen Dondorp, Centre for Clinical Vaccinology and Tropical Medicine, Churchill Hospital, Oxford University, Oxford OX3 7LJ, United Kingdom and Wellcome TrustMahidol University-Oxford Tropical Medicine Research Programme, Faculty of Tropical Medicine, Mahidol University, 420/6 Rajvithi Road, Bangkok, 10400, Thailand, E-mail: arjen@tropmedres.ac.

\section{REFERENCES}

1. WHO, 1999. Counterfeit Drugs. Guidelines for the Development of Measures to Combat Counterfeit Drugs. Available from http:// www.who.int/medicines/publications/counterfeitguidelines/ en/.

2. O'Brien KL, Selanikio JD, Hecdivert C, Placide MF, Louis M, Barr DB, Barr JR, Hospedales CJ, Lewis MJ, Schwartz B, Philen RM, St Victor S, Espindola J, Needham LL, Denerville $\mathrm{K}, 1998$. Epidemic of pediatric deaths from acute renal failure caused by diethylene glycol poisoning. JAMA 279: 1175-1180.

3. Hanif M, Mobarak MR, Ronan A, Rahman D, Donovan JJ, Bennish ML, 1995. Fatal renal-failure caused by diethylene glycol in paracetamol elixir: the Bangladesh epidemic. BMJ 311: 8891.

4. Newton PN, White NJ, Rozendaal JA, Green MD, 2002. Murder by fake drugs: time for international action. BMJ 324: 800-801.

5. Cockburn R, Newton PN, Agyarko EK, Akunyili D, White NJ, 2005. The global threat of counterfeit drugs: why industry and governments must communicate the dangers. PLoS Med 2: 100-106.

6. Newton P, Proux S, Green M, Smithuis F, Rozendaal J, Prakongpan S, Chotivanich K, Mayxay M, Looareesuwan S, Farrar J, 
Nosten F, White NJ, 2001. Fake artesunate in southeast Asia. Lancet 357: 1948-1950.

7. Newton PN, Green M, Fernandez FM, Day NP, White NJ, 2006. Counterfeit anti-infective drugs. Lancet Infect Dis. 6: 602-613.

8. Basco LK, 2004. Molecular epidemiology of malaria in Cameroon. XIX. Quality of antimalarial drugs used for selfmedication. Am J Trop Med Hyg 70: 245-250.

9. Daviss B, 2005. Malaria, science, and social responsibility. Scientist 19: 42-43.

10. White NJ, 2004. Antimalarial drug resistance. J Clin Invest 113: 1084-1092.

11. van Agtmael MA, Eggelte TA, van Boxtel CJ, 1999. Artemisinin drugs in the treatment of malaria: From medicinal herb to registered medication. Trends Pharmacol Sci 20: 199-205.

12. Aldhous P, 2005. Murder by medicine. Nature 434: 132-136.

13. Dondorp AM, Newton PN, Mayxay M, van Damme W, Smithuis FM, Yeung S, Petit A, Lynam AJ, Johnson A, Hien TT, McGready R, Farrar JJ, Looareesuwan S, Day NPJ, Green MD, White NJ, 2004. Fake antimalarials in southeast Asia are a major impediment to malaria control: multinational crosssectional survey on the prevalence of fake antimalarials. Trop Med Int Health 9: 1241-1246.

14. Newton PN, Dondorp A, Green M, Mayxay M, White NJ, 2003. Counterfeit artesunate antimalarials in southeast Asia. Lancet 362: 169.

15. Newton PN, McGready R, Fernandez FM, Green MD, Sunjio M, Bruneton C, Phanouvong S, Millet P, Whitty CJ, Talisuna AO, Proux S, Christophel EM, Malenga G, Singhasivanon P, Bojang K, Kaur H, Palmer K, Day NPJ, Greenwood BM, Nosten F, White NJ, 2006. Manslaughter by fake artesunate in Asia: will Africa be next? PLoS Med 3: 1-4.

16. Green M, Mount DL, Wirtz RA, 2001. Authentication of Artemether, Artesunate and dihydroartemisin antimalarial tablets using a simple colorimetric method. Trop Med Int Health 6: 980-982.

17. Lim CK, Lord G, 2002. Current developments in LC-MS for pharmaceutical analysis. Biol Pharm Bull 25: 547-557.

18. NIST Scientific and Technical Databases, 2002. Available from http://www.nist.gov/srd/nist1a.htm.

19. Vankeirsbilck T, Vercauteren A, Baeyens W, Van der Weken G, Verpoort F, Vergote G, Remon JP, 2002. Applications of Ra- man spectroscopy in pharmaceutical analysis. TrAC Trends Anal Chem 21: 869-877.

20. Massart DL, Vandeginste BG, Buydens LM, De Jong S, Lewi PJ, Smeyers-Verbeke J, 1997. Handbook of Chemometrics and Qualimetrics. Amsterdam: Elsevier.

21. World Health Organization, 2006. What Are Substandard Drugs? Available from http://www.who.int/medicines/services/ counterfeit/faqs/06/en/.

22. Gates P, Kearney G, Jones R, Leadlay P, Staunton J, 1999. Structural elucidation studies of erythromycins by electrospray tandem mass spectrometry. Rapid Commun Mass Spectrom 13. 242-246.

23. Wardrop J, Ficker D, Franklin S, Gorski R, 2000. Determination of erythromycin and related substances in enteric-coated tablet formulations by reversed-phase liquid chromatography. $J$ Pharm Sci 89: 1097-1105.

24. Allen LV Jr, Popovich NG, Ansel HC, Allen LV, 2004. Ansel's Pharmaceutical Dosage Forms and Drug Delivery Systems. Philadelphia: Lippincott Williams and Wilkins.

25. Jähnke RW, Küsters G, 2001. Detecting counterfeit drugsprotection against counterfeit medicines using the GPHFMinilab. Bull Mekong Malaria Forum 8: 118-124.

26. Rozendaal J, 2000. Fake antimalarials circulating in Cambodia. Bull Mekong Malaria Forum 7: 62-68.

27. United States Pharmacopeia, 2006. Fake Malarials Found in Yunnan Province, China. Available from http://www.uspdqi.org pubs/other/FakeAntimalarialsinChina.pdf.

28. Hartl DL, 2004. The origin of malaria: mixed messages from genetic diversity. Nat Rev Microbiol 2: 15-22.

29. Fernandez FM, Cody RB, Green M, Hampton CY, McGready R, Sengaloundeth S, White NJ, Newton PN, 2006. Characterization of solid counterfeit drug samples by desorption electrospray ionization and direct-analysis-in-real-time coupled to time-of-flight mass spectrometry. Chem Med Chem 1: 702-705.

30. Wolff JC, Thomson LA, Eckers C, 2003. Identification of the 'wrong' active pharmaceutical ingredient in a counterfeit Halfan $^{(\mathrm{TM})}$ drug product using accurate mass electrospray ionisation mass spectrometry, accurate mass tandem mass spectrometry and liquid chromatography/mass spectrometry. Rapid Commun Mass Spectrom 17: 215-221. 